\title{
La atención a las madres durante el proceso de parto en algunos servicios de salud de la ciudad de Medellín: un acontecimiento enmarcado en el neoliberalismo y la mercantilización de la vida
}

\author{
Health Care to Mothers during the Delivery in some Health Centers in the City of Medellín: An Event \\ Framed under the Neoliberalism and the CommodiAcation of Life \\ O atendimento às mães durante o processo de nascimento em alguns serviços de saúde da cidade de Medellín: \\ um evento enquadrado no neoliberalismo y a mercantilização da vida
}

\author{
Juan David Arango Urrea ${ }^{\text {a }}$ \\ Universidad de Antioquia, Colombia \\ paterdas@yahoo.es \\ ORCID: http://orcid.org/0000-0002-3919-7256 \\ Diana Patricia Molina Berrío \\ Universidad de Antioquia, Colombia \\ ORCID: http://orcid.org/0000-0002-1352-1089 \\ Cristina María Mejía Merino \\ Universidad de Antioquia, Colombia \\ ORCID: http://orcid.org/0000-0003-2782-9567 \\ Lyda Faneyra Zapata \\ Universidad de Antioquia, Colombia \\ ORCID: http://orcid.org/0000-0002-0354-4068
}

DOI: https://doi.org/10.11144/Javeriana.rgps17-35.amdp Redalyc: http://www.redalyc.org/articulo.oa?id=54557477004

Fecha de recepción: 23 Octubre 2017

Fecha de aprobación: 03 Mayo 2018

Fecha de publicación: 01 Noviembre 2018

\section{Resumen:}

Objetivo: comprender los significados que tiene el personal asistencial sobre la influencia que ejercen el contexto de salud y las condiciones laborales en la atención a las madres durante el proceso de parto, en algunos servicios de salud de la ciudad de Medellín en los años 2015-2016. Metodología: estudio de naturaleza cualitativa, usando la metodología propuesta por la teoría fundamentada (TF) de Anselm Strauss y Julieth Corbin. Resultados y conclusión: las relaciones entre el personal asistencial y las gestantes en proceso de parto están enmarcadas en un macrocontexto, como lo es el Sistema de Salud de Colombia, y una perspectiva neoliberal que mercantiliza la vida. El cierre de muchos servicios de maternidad y algunas condiciones laborales difíciles han afectado las relaciones entre el personal de salud y las madres durante el proceso de parto.

Palabras clave: condiciones de salud, personal de salud, violencia contra la mujer, servicios de salud materna, parto obstétrico, Colombia.

\section{Abstract:}

Objective: To understand the meanings that health care workers have about the influence of the health context and the labor conditions on the health care provided to mothers in their delivery process, in some health centers in the city of Medellín during 2015-2016. Methods: It was a qualitative study using the methodology proposed after Anselm Strauss and Julieth Corbin's Grounded Theory (GT). Results and Conclusion: The relations between the health care workers and the pregnant women during the delivery process are framed under both a macro-context like the Colombian health system and a neoliberal perspective that commodifies the life. The shutdown of many maternity wards and some of the hard labor conditions have impacted the relations between the health care workers and the mothers during the delivery process.

Keywords: health conditions, health care workers, violence against women, maternal health services, obstetrical delivery, Colombia. 
Resumo:

Objetivo: compreender os significados os profissionais da saúde têm sobre a influência do contexto de saúde e das condições laborais na atenção às mães durante o processo de parto, em alguns serviços de saúde da cidade de Medellín nos anos 2015-2016. Metodologia: estudo de natureza qualitativa, a usar a metodologia proposta pela teoria fundamentada (TF) de Anselm Strauss e Julieth Corbin. Resultados e conclusão: as relações entre a equipe assistencial e as gestantes no processo de parto estão enquadradas em um macrocontexto, como é o Sistema de Saúde da Colômbia e uma perspectiva neoliberal que mercantiliza a vida. O fechamento de muito serviço de maternidade e algumas condições laborais difíceis afetaram os relacionamentos entre o pessoal de saúde e as mães durante o processo de parto.

Palavras-chave: condições de saúde, pessoal de saúde, violência contra a mulher, serviços de saúde materna, parto obstétrico, Colômbia.

\section{Introducción}

La salud sexual y la reproducción son elementos esenciales en el proceso vital de los seres humanos y en el desarrollo de los pueblos (1). Garantizar los derechos sexuales y los derechos reproductivos (DS y DR) como los más humanos de todos, y con un enfoque de género, para el ejercicio pleno de la ciudadanía en un marco libre de violencias, igualdad, libertad y autonomía, constituye un objetivo primordial de la salud pública de cualquier país (2). Cuando estos son vulnerados en cualquiera de sus dimensiones, ello supone un elevado costo para los gobiernos, los colectivos y los individuos de cualquier sociedad (3).

En este marco de derechos, el mejoramiento de la salud materna desempeña un papel protagónico. En efecto, la salud de las mujeres gestantes se ha transformado en un indicador muy importante en el desarrollo de las naciones y ha sido puesta en la agenda internacional como uno de los Objetivos para este Milenio (4). Dicho objetivo ha sido retomado por la Organización de las Naciones Unidas (ONU) en la Convención de 2015, donde los objetivos tres y cinco, especialmente, para un desarrollo sostenible apuntan de una manera particular a la disminución de la morbilidad y la mortalidad materna, garantizando al mismo tiempo una vida sana y la promoción del bienestar (5). Al respecto, la Organización Mundial de la salud (OMS) declara que "en 2013, 289.000 mujeres fallecieron durante el embarazo o el parto [...] La mayoría muere porque no se dispone de suficiente personal competente en la prestación de atención de rutina o de emergencia" (6) y, aunque en el 2015 la Convención del Desarrollo Sostenible aceptó que el número de muertes maternas había disminuido, llama la atención acerca de aquellas muertes maternas que todavía hoy se presentan y que para las regiones en desarrollo es 14 veces mayor que en las regiones desarrolladas (5). Una relación desproporcionalmente desigual y al mismo tiempo injusta.

Mejorar la salud materna implica implementar políticas en salud pública que garanticen el bienestar del binomio madre-hijo, especialmente en los momentos más críticos de la gestación, entre los cuales el proceso del parto marca un momento trascendental y decisivo en la vida y la salud de la mujer y del recién nacido (7). Sin embargo, estudios recientes revelan cómo, precisamente, es durante el parto cuando los derechos de la mujer son más vulnerados, transformándose esta en un objeto de violencia por parte del personal asistencial que las atiende en los centros de salud (8). Según un reporte de la OMS del 2014:

En todo el mundo, muchas mujeres sufren un trato irrespetuoso y ofensivo durante el parto en centros de salud, que no solo viola los derechos de las mujeres a una atención respetuosa, sino que también amenaza sus derechos a la vida, la salud, la integridad física y la no discriminación. (8)

Esta problemática parece contener subyacentes dos miradas que se yuxtaponen entre sí y que no pueden excluirse, por el riesgo que supone perder una visión global de dicha realidad y las posibles causas que la determinan.

Por una parte, se encuentra la violencia contra las mujeres en el proceso de gestar y dar a luz una nueva vida, y en la otra cara, se encuentra el personal de salud que en el terreno asistencial puede actuar de acuerdo con escenarios generales tales como la cultura, el modelo médico hegemónico, el contexto del sistema de salud, la segmentación y la especialización de la práctica sanitaria, las condiciones laborales, la precariedad y saturación 
del sistema hospitalario, un recurso humano insuficiente para atender la demanda en maternidad, las políticas institucionales que muchas coartan veces la libertad y el criterio de los profesionales, la atención centrada en la tecnología o, por el contrario, la falta de insumos necesarios para atender a las gestantes y, finalmente, la poca o nula capacitación y formación del personal en temas como el parto humanizado y los DSSR, lo que propicia prácticas de baja calidad en la atención obstétrica, deriva en la negligencia y, como última consecuencia, en expresiones de violencia institucional (9).

También existen situaciones particulares que pueden influir en el trato hacia las gestantes, entre las que se encuentran la formación recibida, el desconocimiento del personal de salud sobre los derechos humanos y los derechos sexuales y reproductivos de la mujer, (9) los propios imaginarios, las demandas y expectativas de las pacientes y sus acompañantes; incluso, asuntos como la inseguridad y el miedo frente a un evento trascendental como lo es el nacimiento (10).

En Medellín, al igual que en Colombia, hay pocos estudios que documenten la atención y el cuidado de las maternas y del recién nacido en las salas de parto, así como las problemáticas que se pueden generar allí como un evento de salud pública importante para la ciudad. Alrededor de las situaciones mencionadas surgen preguntas como: ¿es la violencia obstétrica un evento frecuente en las salas de parto de la ciudad o son casos aislados? Si se presenta, ¿de qué forma se da?, ¿qué factores influyen para que esto suceda en dicho contexto?, ¿el personal asistencial reconoce este tipo de prácticas?, ¿qué explicación da el personal asistencial a la ocurrencia de este fenómeno?

El presente estudio hace parte de un proyecto macro que busca comprender los significados de las gestantes y del personal asistencial en torno al proceso del parto, para de esta manera tener una mirada de conjunto sobre el fenómeno estudiado sin excluir a ninguno de los actores que participan en el proceso. Sin embargo, se aclara que este artículo solo incluirá la visión del personal asistencial. En cuanto a la perspectiva de las madres, se abordará en otro artículo.

Es importante resaltar que en los últimos años el personal asistencial se ha visto inmerso en la dinámica neoliberal que ha llevado a la crisis de la salud, puesto que dicho sistema se basa en una lógica económica y no en la satisfacción de las necesidades reales de las personas, tal y como lo propone Laurell (11). Así, la mercantilización de la salud y de la vida ha estado acompañada del modelo biomédico hegemónico, de la medicalización de la vida, la tecnocratización del cuerpo humano y, finalmente, la flexibilización laboral de los trabajadores de la salud (11). Precisamente en este contexto, el personal asistencial desarrolla la atención a las madres durante su proceso del parto, lo que termina afectando las relaciones entre los profesionales y las madres y ha sido documentado en algunos casos, como el de Brasil (12).

Comprender las experiencias y los significados del personal asistencial en el proceso del parto permitió identificar el estado del reconocimiento de los derechos humanos y de los DS y DR por parte de estos hacia las mujeres. También dio luces acerca de cómo se da la relación entre los profesionales y la gestante y cómo en dicha relación se ponen en juego aspectos señalados por algunos estudios $(9,10,12,13,14)$, como el contexto en salud, la praxis hegemónica de poder y el control simbólico, especialmente desde algunos paradigmas como el biomédico, el contexto y la infraestructura institucional, las condiciones laborales, la in fluencia de los procesos formativos, así como los significados e imaginarios del personal de salud en torno al proceso del parto y a las relaciones con las madres durante la asistencia.

Todo lo anterior permitió, finalmente, proponer miradas y alternativas para que el personal asistencial pudiera contar con escenarios propicios y condiciones laborales justas y dignas que lo orienten en la construcción de un trato humanizado y un servicio integral de la madre. Escenarios que recreen la intimidad del hogar y retornen el papel protagónico a la madre y a sus seres queridos en el acompañamiento de un acontecimiento tan trascendental como lo es el parto. También se evidenció la necesidad, urgente, en cuanto a la formación y actualización continuada de los técnicos y profesionales que participan en estos procesos, especialmente sobre parto humanizado, DS y DR, educación para la salud, calidad y seguridad para la atención y cuidado de la gestante, su bebé y su familia de una manera holística, transdisciplinaria y transpersonal. 


\section{Metodología}

\section{Tipo y diseño de estudio}

La presente investigación fue un estudio de carácter cualitativo para el cual se eligió la teoría fundada, desarrollada por Glaser y posteriormente por Strauss con la ayuda de Julieth Corbin (15). Se eligió la teoría fundada porque como enfoque de diseño metodológico ofrece la posibilidad de pensar la realidad social de una manera sistemática y estudiarla; además, porque su proceso toma muy en serio el lenguaje y las acciones de las personas investigadas (15). De esta forma, la intención del investigador es: "Descubrir el significado profundo de la experiencia vivida por los individuos en términos de sus relaciones con el tiempo, espacio, vivencias e historia personal" (16). Los procedimientos que propone la teoría fundada se enfocan en la comprensión de un fenómeno expresado en su teorización (15).

\section{Participantes y criterios de participación}

Como criterios de participación se tuvieron los siguientes: 1) personal del área asistencial en salud o estudiantes en nivel de residencia en ginecobstetricia; 2) laborar en un servicio clínico (urgencias, hospitalización, sala de partos o cirugía) de una institución de salud de la ciudad de Medellín que atendiera a la gestante en el proceso del parto; 3) tener experiencia asistencial en el área obstétrica como mínimo de tres meses. Por razones de confidencialidad no se nombran las instituciones que participaron del estudio, sin embargo, se aclara que fueron siete, en su mayoría privadas, cinco de tercer nivel de atención y dos de segundo nivel. De cada institución se contó al menos con dos participantes.

\section{Técnicas e instrumentos para la recolección de la información}

Se inició con ocho participantes hasta completar dieciséis. A cada participante se le realizó una entrevista semiestructurada. Se realizaron en total tres fases de recolección de información, cada una de las cuales contó con su instrumento (entrevista semiestructurada), y este se fue modificando a medida que iban emergiendo y se analizaban los datos, así como al terminar cada una de las fases. En la primera fase se realizaron ocho entrevistas, en la segunda fase cuatro y en la última también cuatro. Inicialmente, se hizo un muestreo a conveniencia, teniendo presente las primeras personas que eligieron participar del estudio y utilizando la técnica de bola de nieve, es decir, que los primeros informantes nos llevaron a otros, para terminar en un muestreo teórico orientado a la búsqueda de categorías faltantes definidas desde el inicio o aquellas que emergieron durante todo el proceso de recolección de los datos y que se configuraron como significativas (17). Al final de la última fase, se tuvo en cuenta el concepto de saturación teórica, en el cual "el número de sujetos por cantidad pierde significado, siendo la riqueza y variedad de los datos obtenidos lo que determina la saturación teórica de la muestra" (18).

\section{Análisis de la información}

Las entrevistas fueron grabadas, previo consentimiento informado, y procesadas para su transcripción y posterior análisis en formato electrónico. Después de su transcripción, se ingresaron a la herramienta informática Atlas-ti versión 6.2, cuyo objetivo fue facilitar el proceso de análisis cualitativo de los volúmenes textuales con los que se contaba. 
En cuanto al procesamiento de los datos y de acuerdo con la metodología elegida, se utilizaron técnicas de codificación línea por línea y categorización para el análisis de la información recolectada. Todo el análisis pasó por los diferentes momentos descritos por Strauss y Corbin: el descriptivo, el analítico y el interpretativo, hasta lograr la teorización o comprensión del fenómeno de estudio (15). Este procesamiento y análisis se describirá a continuación más detalladamente. Un total de cuatro investigadores analizaron toda la información y, al terminar cada fase, se llevaron a cabo al menos tres sesiones de discusión y retroalimentación.

Durante el análisis microscópico línea por línea de cada una de las entrevistas, ocho en un primer momento, emergieron en total once categorías descriptivas, las cuales se presentan en la figura1

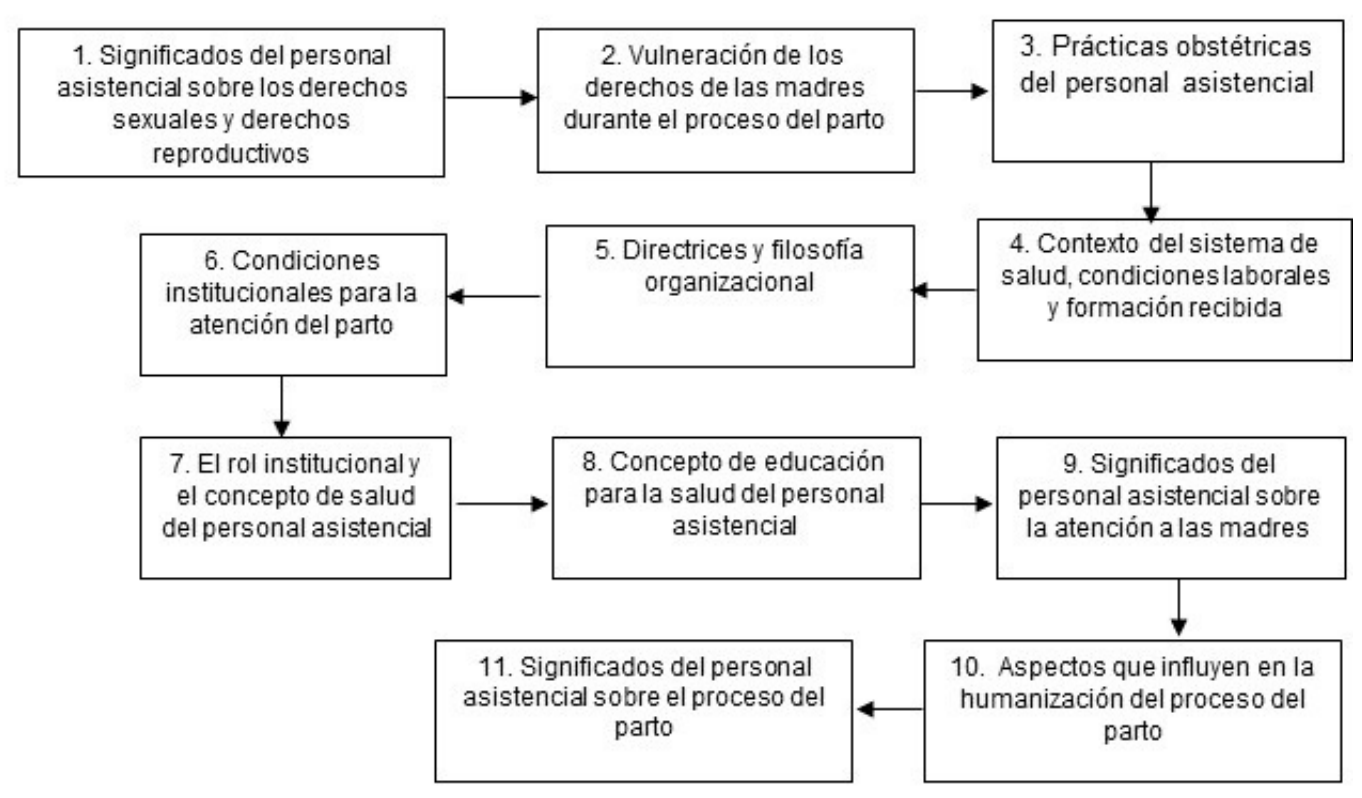

FIGURA 1

Categorías descriptivas resultantes de la codificación abierta

elaboración propia.

Cada una de estas categorías emergió con sus propiedades y dimensiones, las cuales ayudaron a construir la entrevista de la siguiente fase y orientaron el camino hacia la codificación axial, tal y como lo propone la teoría fundamentada. En esta parte del proceso se aplicó el otro instrumento, el cual se orientó a buscar las relaciones existentes entre las diferentes categorías descriptivas. Durante esta fase se llevaron a cabo cuatro entrevistas, las cuales se codificaron de nuevo. Finalmente, de una manera analítica, se relacionaron las categorías y las subcategorías, tratando de agrupar mejor los fenómenos relacionados y que presentaron mayor fuerza. Terminado este momento, las categorías descriptivas se fusionaron de acuerdo con las relaciones encontradas, y quedaron así ocho categorías analíticas, las cuales se describen en la tabla 1. 
TABLA 1

Categorías analíticas resultantes de la codificación axial

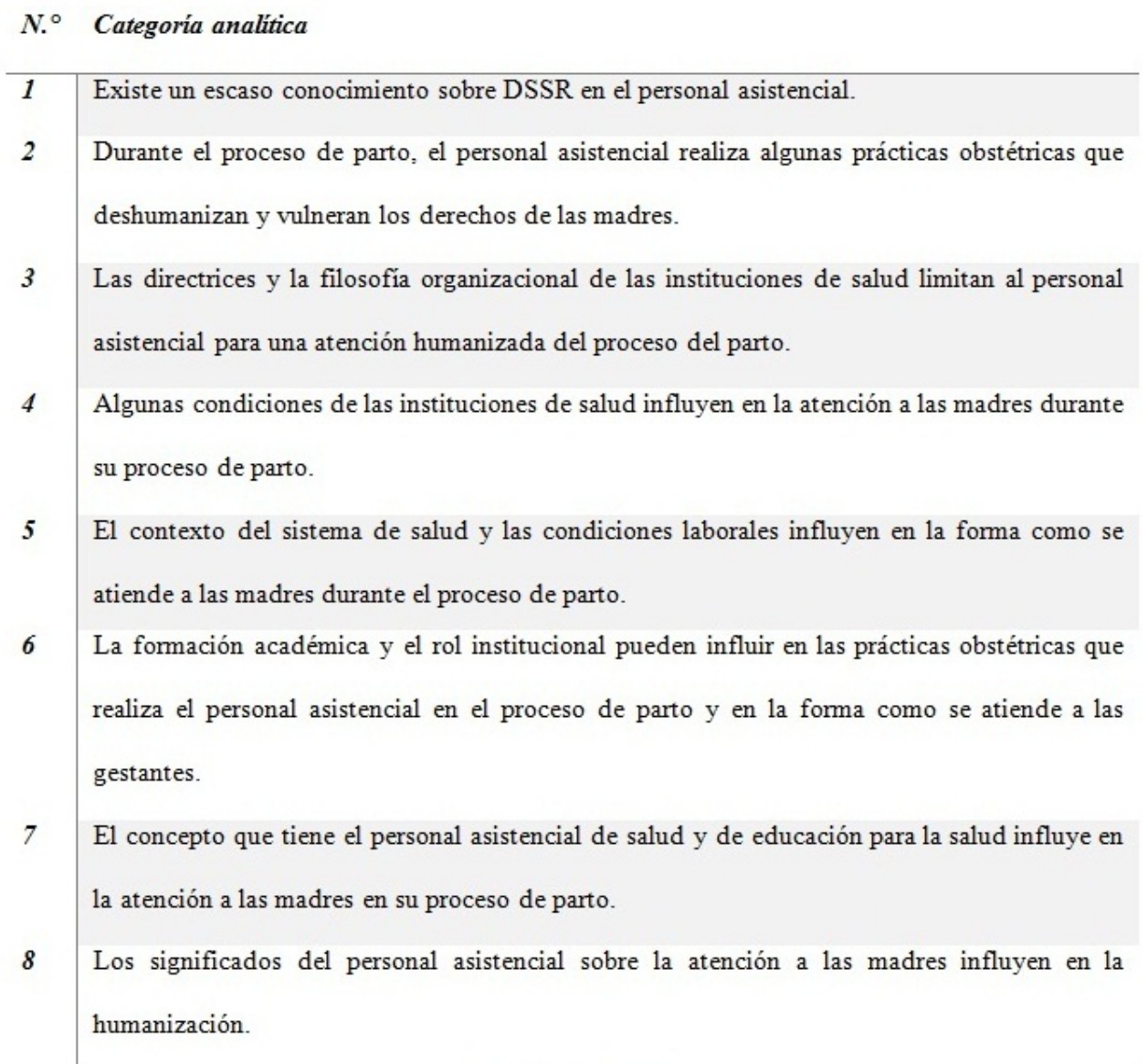

Fuente: elaboración propia.

Por último, se realizó un grupo de cuatro entrevistas, con el fin de validar las últimas relaciones encontradas, es decir, que los discursos previamente escuchados por el investigador se hubieran interpretado de manera adecuada, para finalmente integrar y refinar la teoría. A lo largo de todo el estudio emergió un total de 1152 códigos, cada uno resultado del análisis microscópico, tal y como lo propone la teoría fundada. Después de tener las categorías totalmente saturadas, se renombraron dichas categorías, con el fin de lograr la mayor integración de toda la información recolectada a lo largo de cada una de las fases del proceso de análisis y comprender de una manera lógica y coherente las palabras de cada uno de los participantes. En esta última parte del proceso quedaron 7 categorías interpretativas, con las 36 subcategorías respectivas que las sustentan. Es importante reconocer que, como limitaciones de este estudio, no se contó con una gran participación de especialistas en ginecobstetricia, como se hubiese querido; en efecto, estos fueron los participantes más limitados, puesto que muchas veces es difícil contactarlos o que dispongan del tiempo para las entrevistas. Sería muy importante llevar a cabo investigaciones donde se cuente con un amplio grupo de especialistas en ginecobstetricia y de esta manera poder comprender mejor lo que ellos piensan y sus experiencias en torno a la maternidad y el proceso de parto. 
Este artículo presenta solamente uno de los resultados centrales de este estudio, el cual está relacionado de manera directa con el macrocontexto en el cual el personal asistencial ejerce la atención del proceso de parto. Es importante aclarar que el fenómeno central presenta una gran riqueza de testimonios que apoyan la comprensión de este fenómeno concreto. El fenómeno central ha sido nombrado por los autores de la siguiente manera: "El contexto del sistema de salud y las condiciones laborales influyen en la forma como se atiende a las madres durante el proceso de parto". La figura 2 presentará la matriz que contiene la explicación interpretativa del fenómeno, la cual se sustenta en cuatro categorías que apoyan su construcción y permiten comprender de una manera más amplia la interpretación del investigador. Cada una de estas categorías contiene los fragmentos y los testimonios de los participantes, así como la discusión y la reflexión que hace el investigador en torno a ellas. Es importante resaltar que el fenómeno que se expone a continuación habla solo del contexto a nivel macro, ya que otros resultados de este proceso investigativo están orientados más a la comprensión de los microcontextos. Sin embargo, se reconoce que el macrocontexto influencia y en algunos casos determina las prácticas que se realizan en otros niveles como el hospitalario o el educativo, por lo que se hace fundamental comprender en primera instancia lo que sucede en un nivel superior, como lo es el sistema de salud y el sistema laboral de Colombia. Cada una de estas categorías aporta desde su propia visión a la compresión global del problema.

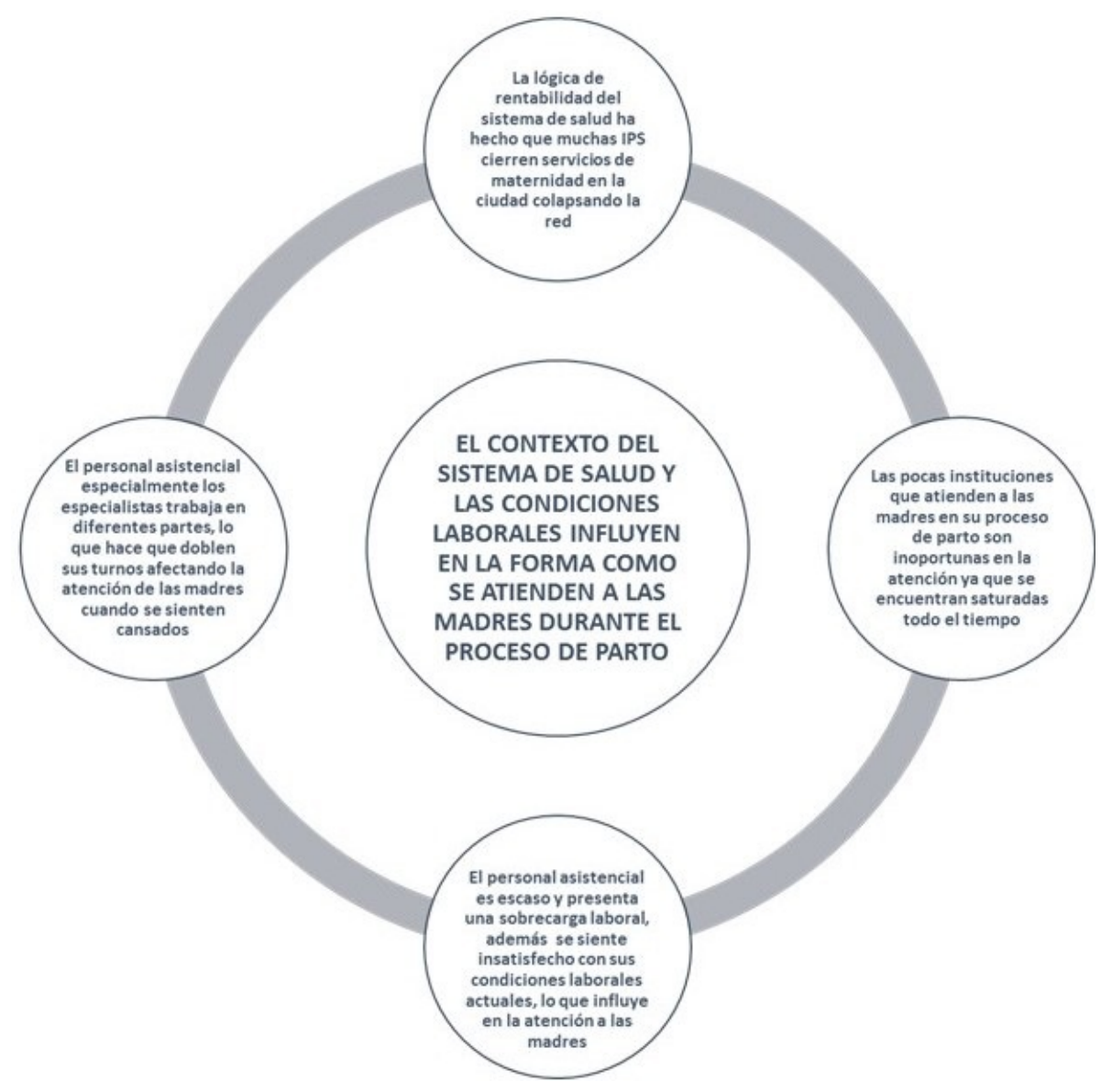

FIGURA 2

Modelo explicativo del fenómeno central con las cuatro categorías que lo sustentan 


\section{Resultados y discusión}

Los salubristas se enfrentan a diario a una práctica en salud que está atravesada por múltiples contextos unos más adversos que otros-, los cuales influyen en gran medida en la forma como se presta la asistencia. Las características de la época contemporánea, los reacomodos globales, la consolidación de nuevos bloques de poder y las nuevas reglas de juego económico terminan por afectar los escenarios en los que se desarrolla la salud pública, (19) es decir, sobre aquellos lugares en que las personas viven, aman, aprenden, trabajan y juegan (20).

La práctica del personal asistencial de Colombia, y concretamente el de Medellín, no es ajena a estos reacomodos globales y a las políticas económicas actuales. Para Pérez Lugo, la práctica en salud en América Latina continúa teniendo una visión sumamente asistencialista, que además se desarrolla en medio de un sistema de administración deficiente, puesto que presenta dificultades en su planificación, organización y control de los recursos (19). Finalmente, Lugo reconoce que nuestro sistema está atravesado por innumerables problemas de cobertura, accesibilidad y legitimidad que terminan afectando la salud de las poblaciones, la situación laboral de los trabajadores de la salud y la estabilidad y credibilidad del sistema político existente (19).

La categoría presentada responde precisamente a un macrocontexto en el que la forma como se atiende a las madres en su proceso de parto se origina en gran medida del sistema de salud establecido y de las condiciones laborales en las que el personal asistencial ejerce su práctica, sin ser por supuesto las únicas condiciones que determinan dicha atención.

\section{La lógica de rentabilidad de la salud: un sistema extremadamente codicioso}

En un primer lugar, se puede afirmar que existe una lógica de rentabilidad del sistema, lo cual ha llevado a que muchas IPS cierren sus servicios de maternidad y a que la red materno-infantil de la ciudad de Medellín colapse. En efecto, no es extraño que el sistema de salud de Colombia este atravesando en los últimos años una crisis que ha terminado por impactar en las condiciones en las que los profesionales y técnicos de salud ejercen su práctica. Rubio León en su estudio sobre el SGSS de Colombia encontró al respecto:

El sistema de salud le quitó la potestad al médico y se la dio a las EPS para mover plata, para mover masas y plata [...] Aquí no se puede hacer nada, usted tiene las manos atadas [...] Todo esto va generando descontento en los pacientes y un profundo desánimo en el personal asistencial. (21)

Es precisamente en un contexto adverso y de descontento en que el personal asistencial de la red maternoinfantil de la ciudad de Medellín ejerce su práctica. A propósito de ello, uno de los participantes de este estudio relató lo siguiente:

Considero con respecto a maternidad que nos falta mucho en la ciudad, nos falta mucho, mucho, - pero uno entiende — las condiciones laborales en este momento no son las mejores, el gremio de ginecobstetra vive cansado, hay muchísima demanda, la tasa de natalidad sigue en aumento, las mamás se siguen embarazando, los servicios de obstetricia siguen colapsados. (E4EPCP154)

El primer significado que tiene el personal asistencial acerca de las relaciones que establece con las madres durante su proceso de parto es que la atención durante dicho proceso se debe realizar en un contexto de salud que está cada vez más influido por una lógica de rentabilidad y mercantilización de la vida. Esta lógica ha llevado a que muchas IPS cierren sus servicios de maternidad, alegando siempre que es un servicio improductivo y poco rentable, lo que ha conllevado el colapso de la red materno-infantil.

Así, algunos artículos de la prensa escrita $(22,23)$ han mostrado un panorama poco alentador acerca de los cierres del servicio de maternidad de la ciudad: 
Por dinero o por amor: Los especialistas de la Clínica León XIII creen que el cierre obedece a una motivación distinta a la expresada oficialmente por la IPS. Según Mercado, era de público conocimiento entre el personal de la Clínica que el servicio de maternidad no era el negocio más atractivo para las directivas del hospital. (22)

Demora en los tiempos de atención y colapso en la red hospitalaria que sirve a las maternas de Medellín denuncian algunas asociaciones médicas de Ginecobstetras, quienes miran con preocupación cómo tres hospitales (el General, la Clínica Universitaria Bolivariana y la del Prado) están concentrando la mayoría de los servicios que otras instituciones han dejado de prestar. (23)

Esta lógica de rentabilidad y de convertir la vida en un negocio ha llevado, por supuesto, a la saturación de la red, pero también ha hecho que las pocas instituciones que queden abiertas colapsen, se vuelvan inoportunas en la atención y sobrecarguen laboralmente al personal asistencial, lo que en últimas lleva al punto central y es, precisamente, la afectación de la atención que se presta a las madres en su proceso de parto. No en vano, aumentan las quejas de las madres, las largas esperas y las complicaciones alrededor de este proceso. Ante esta lógica neoliberal, uno de los participantes refería:

[...] Pero resulta que en este momento en la ciudad estamos en una atención critica a las maternas, muchas instituciones han decidido cerrar sus servicios de maternidad por el bajo costo que equivale la atención de un parto. (E4EPCP5

En el estudio realizado por el diario El Tiempo se menciona que algunas esperas por parte de las madres para la atención son de hasta doce horas, (23) sin embargo, en esta investigación se ha encontrado que incluso hay mamás que pueden pasar hasta dieciocho horas esperando para ser atendidas:

[...] Entonces la clínica se mantiene llena y no damos abasto y el sobrecupo es cada vez mayor, la atención en urgencias es cada vez más demorada, el triage es muy demorado, pueden transcurrir hasta 18 horas para una atención. (E4EPCP54)

Este es entonces el sistema de salud que se hoy en día presenta al personal asistencial que atiende los procesos de parto de nuestras madres. Un sistema que como señala Santos es "Goloso hasta el extremo" (24) y que termina por afectar la atención en un momento tan importante en la vida de todo ser humano como es el nacimiento. Aquí todos los protagonistas: la madre y su hijo, el personal médico y de enfermería y, por supuesto, todos aquellos que rodean el proceso de parto se ven enfrentados a diario a vivir situaciones injustas, como las largas esperas, la deshumanización de la atención y, por qué no, la frustración de un momento que debería ser maravilloso para quienes lo viven:

[...] Entonces la carga se hace muy pesada, me siento, me siento a veces frustrada, realmente me siento frustrada porque siento como que estoy perdiendo conocimientos en muchas cosas, conocimientos, y entender porque a la paciente le está ocurriendo esto y no esto $[\ldots]$ (E4EPCP73)

En efecto, el sistema de salud colombiano, en el cual el Estado cada vez está menos presente, continúa llevando a que se propague la incertidumbre, a que muchas IPS cierren servicios que son, de acuerdo con el modelo instaurado, poco rentables; a que se quiebren otros tantos y a que finalmente se instaure un sistema que no tiene ningún control, y sumado a esto una gran corrupción. Santos hace una reflexión contundente relacionada con estas problemáticas:

[...] El discurso que oímos todos los días para hacernos creer que debe haber menos Estado se vale de esa mencionada porosidad, pero su base esencial es el hecho de que los conductores de la globalización necesitan un Estado flexible a sus intereses. Las privatizaciones son la muestra de que el capital se volvió devorador, goloso al extremo, siempre exigiendo más, queriéndolo todo. En verdad, la perversidad deja de manifestarse por hechos aislados, para establecerse como un sistema. El otro, sea empresa, institución o individuo aparece como un obstáculo para la realización de los fines de cada uno y debe ser removido. Se derivan de ahí la celebración de los egoísmos, la propagación de los narcisismos, la banalidad de la guerra de todos contra todos y la difusión de otro subproducto de la competitividad, es decir la corrupción. (24)

La atención se ve influida por un sistema de salud que no posibilita la humanización y fragmenta el cuidado de las gestantes en su proceso de parto. La poca inversión y el olvido por parte del Estado crean un círculo 
vicioso en el que a las aseguradoras les interesa invertir menos en la maternidad y las IPS terminan cerrando este tipo de servicios:

[...] la atención del parto es supremamente regalada, barata y pagan muy poquito, las EPS no le invierten, entonces es muy duro para las instituciones prestar los servicios y bueno [...] las personas hacen lo que se puede como de acuerdo al monto de pacientes que ingresan todos los días. (E4EPCP155)

Cada vez es más notorio el descontento del personal asistencial y el de las gestantes, quienes continuamente reclaman una atención más oportuna y humanizada. Sin embargo, los resultados de esta investigación reafirman que el modelo dominante sigue siendo el económico, y quedan subsumidas las necesidades de la población, todavía más cuando las personas cursan por un proceso de mayor vulnerabilidad como lo es la gestación. El momento histórico que atraviesa nuestro país está marcado por la mercantilización de la vida y de la salud de los ciudadanos. Frente a dicha mercantilización, Laurell sostiene:

El enfoque dominante de la política de salud: Ha sido el económico y no el de satisfacer las necesidades de salud de la población. Ahora la cobertura universal es una exigencia de los ciudadanos a sus gobiernos para favorecer la cohesión social. $(11)$

Es importante que los salubristas reconozcan que todas estas situaciones no son fortuitas, incluida la reforma de salud de Colombia de 1993. Este contexto proviene en gran medida de organismos multilaterales y de sus orientaciones. Una de ellas y la que tal vez más ha influido en las reformas en salud de las Américas es la de "invertir en salud", propuesta por el Banco Mundial (25). Invertir en salud tiene un encubierto, pero sugestivo trasfondo: la mercantilización de la salud, la desprotección del Estado, el traslado de los costos a las familias, la privatización de los servicios y todo un engranaje de perversidades aún más excluyentes: reproducción de los paseos de la muerte, sociedades enfermas y territorios sin esperanza. Esta es tan solo una parte de la radiografía a la que se ven enfrentados los salubristas en la actualidad.

De esta manera, queda en evidencia una reforma ineficiente e ineficaz, pobre en transformación social, incapaz de superar la fragmentación que provenía del sistema anterior, y que induce, por el contrario, a nuevas fragmentaciones, perversas y excluyentes, tal y como lo expresa Almeida:

El principal resultado de esos procesos ha sido que las reformas no fueron capaces de superar la segmentación y la fragmentación de los sistemas de atención, muchas veces introduciendo nuevas segmentaciones perversas en las ya existentes estructurales. (26)

La saturación de la red y de las clínicas y hospitales conlleva una sobrecarga del personal asistencial y un descontento generalizado que hace a la práctica obstétrica aún más difícil. Este es otro microcontexto en el que se desenvuelve la práctica del personal asistencial y en el que se tejen las relaciones con las gestantes.

\section{Instituciones de salud saturadas e inoportunas en la atención}

El cierre de los servicios de maternidad y el colapso de la red ha llevado, por consiguiente, a que las pocas instituciones que en la ciudad atienden gestantes se encuentren casi siempre en sobrecupo y, por lo tanto, se vuelvan inoportunas para la atención a las madres:

[...] Pero con respecto al número de camas como tal, para albergar tantas pacientes que ingresan a la institución de toda la ciudad de Medellín no damos abasto, o sea la cantidad de maternas que ingresan es demasiado y tienen que esperar mucho tiempo para ser atendidas, entonces no damos abasto en hospitalización con las pacientes que ingresan. (E4EPCP50)

Es importante mencionar que el personal asistencial se encuentra muy preocupado con esta situación. Los participantes han referido en sus testimonios que cada día el volumen de maternas aumenta, y la problemática, en vez de solucionarse, ha ido empeorando debido a que las instituciones de salud no están interesadas en 
abrir nuevos servicios de ginecobstetricia, en tanto que los pocos que quedan, como se ha mencionado, poco a poco han ido cerrando.

La preocupación radica básicamente en la inoportunidad en la atención, que no solo afecta los indicadores institucionales, lo cual sería el problema menor de acuerdo con los participantes, sino también el riesgo al que someten a las madres con la espera para ser atendidas, pues todos coinciden en que la atención a la gestante y a su hijo es demasiado crítica, ya que en cualquier momento su bienestar se puede ver comprometido:

[...] Porque un trabajo de parto es algo muy incierto, mientras una mamá puede llegar bien, en cualquier momento se puede complicar de la manera más sencilla. (E14AECB28)

La saturación de la red y el consecuente colapso institucional llevan a que diferentes servicios internos de las instituciones de salud se encuentren continuamente abarrotados, en busca de una atención digna y humanizada. Desde las salas de espera, donde muchas de las gestantes incluso logran avanzar en su trabajo de parto, soportando el dolor y la incomodidad propia de este lugar, las salas de hospitalización, donde muchas veces no alcanza el número de camas para cubrir la población materna, y, finalmente, las salas de parto, donde se identifica la necesidad de acelerar los procesos a través de la inducción con oxitocina sintética y las altas tempranas. Todas estas situaciones son identificadas por el personal asistencial como una sumamente problemáticas, en las que se violan algunos derechos de las madres y conllevan una insatisfacción generalizada de su proceso de atención durante el trabajo de parto, el parto y el posparto.

A todas las gestantes se les pone oxitocina para acelerar su proceso de parto. (E6GOCB56)

Para Odent, la oxitocina desempeña un papel importante en muchos episodios de la vida sexual y reproductiva y, además, en las relaciones que se establecen entre la madre y su bebé:

Conocemos el rol que juega la oxitocina en la capacidad de establecer el cruce de miradas, de interpretar los mensajes faciales y de estar en situación de confianza. En otras palabras, comprendemos el rol esencial del sistema de la oxitocina en los procesos de socialización. (27)

De acuerdo con estas palabras de Odent, todavía no dimensionamos el daño que estamos causando al proceso de socialización humana. Una pregunta surge respecto a esta situación: ¿en un futuro, los humanos no seremos capaces de producir el cóctel de hormonas del amor debido a un daño en nuestro sistema de producción? ¿Habrá entonces que inyectarse todos los días un compuesto de oxitocina para poder amar? ¿Qué pasará con los procesos de socialización humanos? Todavía no tenemos la respuesta. La invitación ahora es a comprender mejor este sistema de hormonas del amor y a intentar proteger las lecciones que nos da la fisiología humana:

Se tarda diez minutos en aprender a utilizar el misoprostol. Tomará décadas neutralizar los profundos condicionamientos culturales de milenios y asimilar el concepto de hormona del amor. (27)

Todo esto revela, al mismo tiempo, modelos hegemónicos que atraviesan la vida de los profesionales, incluso de una manera muy sutil, con un enmascaramiento de la realidad, como dice Bourdieu ,que lleva a los seres humanos a formar ese habitus (28). Es decir, prácticas como la medicalización de la vida se rutinizan, se transforman en un hábito en el que la vida queda relegada y muchas formas de violencia se enmascaran.

En consonancia con lo anterior, es posible afirmar que el colapso de los servicios de maternidad y la saturación de la red en la atención a las gestantes durante su proceso de parto traen consigo consecuencias aún más nefastas que la misma inoportunidad, pues se termina acelerando los procesos y ejerciendo una atención análoga a una maquinaria, de una fábrica productora de bebes en un modelo que reconfigura continuamente la economía de mercado. Es decir, las instituciones y los profesionales recrean continuamente el círculo vicioso de la producción en masa, se desligan de la calidad y la humanización que deberían impregnar este acontecimiento en la vida de toda gestante, y ello trae como consecuencia la despersonalización en la atención y la recreación de un ambiente desagradable y violento para el parto: 
[...] Entonces empezamos a saltarnos algunos protocolos de procedimientos, entonces nos saltamos contarle a la mamá estos y estos son tus derechos o podés pedirnos tal cosa, porque se convierte en algo rutinario y en términos sonará muy feo, a ganancias y productivo cierto, estamos en una maquinaria. (E3IQCP11)

De acuerdo con lo anterior, autores como Martín encuentran un símil entre la producción capitalista y la producción de niños. Este autor señala cómo existen grandes similitudes entre operarios industriales y mujeres parturientas: "Desde que el trabajo de parto fue definido como trabajo mecánico realizado por el útero, el nacimiento fue visto como reproducción de bienes" (29). De esta manera, en el proceso de parto, llamado también trabajo de parto, puesto que es semejante a un sistema de producción, el personal asistencial controla el trabajo de las mujeres (gestantes) y de las máquinas (sus úteros) con el fin de obtener el producto fetal (su bebé).

A este respecto, García-Jordá et al., en una investigación revelan cómo el nacimiento es abordado siempre desde una perspectiva medicalizada, con uso rutinario de intervenciones médicas que roban todo protagonismo y poder de decisión a la gestante y su familia, lo que se constituye en una forma de violencia física y de género (30). Los autores concluyen la necesidad de espacios propicios para el nacimiento, donde no impere la medicalización, tranquilos y propiciadores de gestantes y familias protagonistas del nacimiento de sus bebes (30).

\section{La sobrecarga y el estrés de los profesionales: su influencia en la atención a las madres}

Trabajar con gestantes en proceso de parto es una labor que requiere detenimiento, atención y paciencia, es decir, que la espera no es solo de la madre, sino también del personal asistencial que está expectante para el proceso del nacimiento. Por ello, la atención a la madre en proceso de parto demanda no solo personal asistencial capacitado, sino también suficiente para que tanto la gestante como su bebé estén bien cuidados en todo momento.

En la actualidad, el contexto de salud del país, y más concretamente el de la ciudad de Medellín, ha hecho que aumente la demanda de atención de mujeres en proceso de parto y que además estas sean atendidas por personal asistencial que se torna escaso respecto al aumento de las solicitudes diarias de atención. Todo esto ha llevado a que los profesionales y técnicos de la salud que atienden a las madres presenten una sobrecarga de trabajo extenuante y desmotivadora:

[...] Yo recuerdo que cuando era residente, se atendían en promedio 200 partos al mes en el servicio donde trabajaba y ahora que trabajo en el mismo servicio tenemos un tope de 700 partos con la misma gente, con el mismo número de camas [...] (E6GOCB37)

Se evidencia que una de las condiciones institucionales que limitan la atención humanizada del proceso de parto está relacionada con la contratación del personal, puesto que dicha contratación no es suficiente para prestar una atención adecuada a las gestantes. Los participantes manifestaron que el proceso de parto es crítico y requiere un sinnúmero de cuidados; sin embargo, y a pesar de lo crítico de la situación de la materna durante esta etapa de la gestación, no solo desde la parte fisiológica, sino también por la incertidumbre, los temores y una gran cantidad de dudas que tienen las madres, las instituciones de la ciudad no contratan el suficiente personal que ello demanda, lo que termina afectando la atención y la humanización del proceso:

Hay momentos en que los servicios de ginecobstetricia colapsan, porque están a reventar y fuera de eso con el personal con el que se cuenta no es suficiente. (E3IQCP50)

Lo cierto de este asunto y que a veces se enmascara, o en el peor de los casos se naturaliza, es que la atención a las madres se ve limitada porque el personal asistencial no va a poder satisfacer las expectativas y las necesidades de todas las gestantes que tiene bajo su cuidado: 
[...] porque no es lo mismo una sola persona atender a cinco, si la carga laboral es muy dura, eso va a afectar directamente a la materna y ella va a llevar la peor parte por la falta de personal, tanto ginecólogo, como auxiliar y como enfermeras. (E1AECR47)

La sobrecarga laboral puede llevar a episodios de insatisfacción o estrés por parte del personal, lo que termina muy probablemente deshumanizando la atención de las madres. Bermejo, quien ha trabajado ampliamente la humanización de la salud, refiere al respecto:

[...] La enfermedad y otras situaciones de vulnerabilidad hace a la persona sentirse desvalida, por lo que necesita un sistema sanitario lo más humano posible. (31)

Es claro que nuestro sistema de salud actual, y el microcontexto laboral en que se desenvuelve el personal asistencial, muchas veces no están dispuestos para ser lo más humanizados posible, más aún cuando la atención se reduce muchas veces a la mercantilización de los servicios de salud y cuando una gestante encuentra un personal cansado y fatigado:

[...] o sea por tanta carga laboral y por tantas altas, ingreso y egresos por tanta cosa administrativa a veces uno se va olvidando como del ser humano que hay allá con necesidades, entonces esta parte me tiene un poco triste, pero es eso solamente. (E4EPCP74)

De esta manera, se afecta aún más la atención a las madres y se deshumaniza el proceso del parto. Todo un engranaje muchas veces perverso que termina afectando el momento maravilloso del nacer. La propuesta del salubrista debe ser entonces una propuesta de atención digna, humanizada, creativa y realmente cuidadora del proceso de parto, que revitalice las relaciones entre el personal asistencial y las gestantes y lleve a cabo lo que Bermejo llama:

Escuchar la parte escondida del Iceberg, es decir, los miedos, los temores, el sentimiento de indefensión, la confianza y la ilusión que palpitan en lo más íntimo del corazón. (31)

Hay entonces un llamado fuerte por un sistema de salud más justo y unas condiciones laborales mejores y con un talento humano suficiente para atender a las gestantes de la ciudad y del país durante su proceso de parto, de una manera oportuna, digna y justa. Porque lo que se está viendo en la actualidad, es lo que relata el siguiente testimonio:

[...] entonces no se está tomando el tiempo, no se está haciendo como una atención personalizada por decirlo así, se está haciendo todo como caiga, como entre más rápido se vaya mejor, porque así queda la habitación libre, porque si queda la sala de partos sola [...] (E14AECB20) [

Definitivamente, el contexto en el que se atiende a las gestantes precisa hoy más que nunca un viraje, una reforma desde el corazón de la estructura que, por supuesto, influya de manera positiva en todos los microcontextos en los que el personal asistencial ejerce su práctica. Tal vez esta sea la revolución que necesite la atención del momento maravilloso de dar a luz un nuevo ser.

La principal razón por la que el personal se siente insatisfecho es la sobrecarga laboral, situación que influye directamente en la atención a las madres durante su trabajo de parto, parto y posparto. Los participantes de esta investigación coincidieron en que existe un exceso de trabajo en las salas de ginecobstetricia, lo que termina afectando la forma como se atiende a las maternas y aumenta el riesgo de que estas se compliquen:

En la institución trabajamos con las uñas, digo yo con las uñas porque el exceso laboral es tanto que pueden ocurrir mil cosas o sea la paciente se puede complicar en un segundo. (E4EPCP76)

Otra razón por la cual el personal asistencial se siente insatisfecho es la remuneración salarial, situación que algunos participantes manifestaron es desmotivadora, sobre todo cuando se compara con el exceso de trabajo al que se ven sometidos en su práctica diaria con las maternas:

[...] pero pienso que no es la remuneración suficiente para todo el trabajo que nos toca hacer en realidad. (E8GOSC37) 
Todo lo anterior, aunado a la saturación de la red, afecta profundamente la relación que se establece entre las madres en proceso de parto, su familia y estos con el personal asistencial. Hoy más que nunca se precisa de un personal suficiente, capacitado y con unas condiciones laborales dignas y justas para que se puedan generar contextos de atención humanizados y abocados a un cuidado esencial del nacimiento. Si bien la humanización del parto no deriva de manera exclusiva de estos asuntos, esta investigación encontró que influyen de modo sustancial en los procesos de atención a las madres que se llevan a diario en las instituciones de salud de la ciudad.

\section{Cansancio y multiplicidad de empleos del personal asistencial: un círculo vicioso}

Sumado a esta insatisfacción y sobrecarga laboral e incluso a las necesidades de talento humano que presentan las instituciones, gran parte del personal asistencial, especialmente los especialistas, opta por trabajar en diferentes sitios, lo que aumenta la carga y el cansancio del personal:

[...] pues cada quien tiene factores distintos, algunos por factores económicos, entonces cada uno se dobla u hace turno en otras instituciones [...] trabajan mucho de noche, llegan trasnochados hacer un turno y no es lo mismo tener un especialista haciendo las horas que debe hacer, como también lo vemos en personal de enfermería, no es igual la atención que cuando se está descansado [...] (E15MGCS25) [

Esta multiplicidad de trabajos y las largas jornadas laborales, que algunas veces se extienden más de 12 horas, terminan afectando la atención y el cuidado de las madres durante su proceso de parto. De esta manera, tanto el personal médico como el de enfermería, que se halla inmerso en esta problemática, se dedica más a actividades administrativas, como diligenciar la historia clínica de la madre, ordenar medicamentos y exámenes de laboratorio, realizar un kárdex de enfermería y verificar que se cumplan las ordenes médicas. Por el contrario, la labor de estar con la paciente, dialogar con ella, y por supuesto su cuidado, va a quedar relegada:

[...] entonces si ellos vienen, nos pueden llamar y decirnos [...] me tardo unos minuticos más en llegar, tenía noche en otra institución o me voy porque tengo noche en otra institución, o corrido en otro lado, entonces van a llegar cansados, con una sobrecarga laboral, se van a dedicar más a su parte administrativa, no van a estar de lleno con la paciente. (E13EPCR26)

Esta situación trae consigo un descuido de la madre, de su bebe y de su entorno inmediato, en el que se encuentra su familia. Para Boff, el rol cuidador es mucho más que un acto en momento puntual; es una actitud constante que exige responsabilidad y preocupación por el otro, un compromiso que va más allá de un momento de la atención (32), y exige, además, ver al otro como el otro y no como lo otro, es decir, como alguien que nos importa, sobre todo si está oprimido o pasa por un momento de vulnerabilidad:

Aquí encontramos el lugar del nacimiento de la ética que reside en esta relación de responsabilidad frente al rostro del otro, en especial del "más otro" que es el oprimido. En la acogida o en el rechazo, en la alianza o la hostilidad hacia el rostro del otro, se establecen las relaciones más primarias del ser humano y se deciden las tendencias de dominación o de cooperación. (32)

Ciertamente, en el paradigma biomédico dominante la tendencia es de dominación hacia el otro (33), que muchas veces es concebido como lo otro. En este caso concreto, las madres son vistas como un objeto, cosificadas y miradas solo desde la perspectiva de un gran útero que lleva un producto fetal (34). Estos resultados hacen un llamado fehaciente, tanto a las instituciones de salud como a quienes hacen las políticas públicas en salud, a velar por que existan profesionales de la salud realmente cuidadores del proceso del parto. Como se ha dicho, cuidar va más allá que un procedimiento médico, instrumental o enfermerístico, reclama tiempo, responsabilidad y compromiso por parte de quienes brindan atención en salud:

Yo considero que en un trabajo de parto debería ser una situación similar, una persona que te esté alentando, que te tome la mano. Yo recuerdo particularmente una paciente que me decía que le diera la mano y yo le di la mano y ella se sentía tranquila porque yo le estaba tomando la mano, entonces son cosas que no te quitan nada en lo personal y que pueden ser muy gratificantes. (E3IQCP61) 
Se puede evidenciar cómo a lo largo de estos resultados hay un llamado imperante al personal asistencial para que se apropie cada vez más de una atención del proceso del parto totalmente humanística, donde por fin se logre poner un freno a la tecnocratización del cuerpo de la mujer, a la medicalización inconsciente y a la mercantilización de un tesoro inalienable como lo es la salud. Ese es el auténtico y revolucionario llamado a los profesionales y técnicos de la salud de nuestro tiempo, a ser capaces de combatir y luchar en contra de las políticas de intereses de las élites de poder y que desde cada espacio donde se gesta la salud de los seres humanos se pueda demostrar que es posible una atención humanizada y al servicio de la vida.

Finalmente, es posible afirmar que el desarrollo de este fenómeno con sus categorías ha mostrado cómo, efectivamente, la atención a las madres está atravesada por un contexto en el que el sistema de salud y las condiciones laborales influyen de manera significativa en la atención que se brinda a las madres durante su proceso de parto. Es importante que los salubristas continúen reflexionando en torno a estos aspectos y que realicen intervenciones innovadoras y contundentes, puesto que nuestro modelo de salud se ha transformado en un prototipo fehaciente del modelo neoliberal, en el que la mercantilización de la vida ha hecho su nido, y, por lo tanto, continuamos poniendo la salud y el comienzo de la vida en manos de un sistema de "mercaderes" cuyo principal objetivo es la compraventa de servicios, que relega en todo momento el valor inalienable de la mujer y su hijo.

\section{Conclusiones}

Las relaciones entre el personal asistencial y las gestantes en proceso de parto están enmarcadas en un macrocontexto, como lo es el sistema de salud de Colombia, y una perspectiva neoliberal en la que se mercantiliza la vida. Este contexto ha llevado a que se cierren muchos servicios de maternidad de la ciudad de Medellín, a que colapse la red materno-infantil y a que los profesionales deban ejercer su práctica diaria en instituciones saturadas respecto a la atención a las madres.

- El colapso de la red materno-infantil de la ciudad de Medellín ha llevado a que las gestantes en proceso de parto deban esperar muchas horas para ser atendidas por el personal asistencial y luego de su ingreso al servicio de maternidad deban realizar su trabajo de parto en condiciones de incomodidad, poca intimidad, medidas para agilizar el parto y altas sumamente tempranas.

- Las altas demandas de maternas en la ciudad de Medellín, que superan a las instituciones que las atienden, han llevado a una sobrecarga laboral del personal asistencial, lo que afecta las relaciones que se tejen entre estos y las madres y termina muchas veces en una deshumanización de la atención.

\section{Recomendaciones}

Favorecer y aportar a la creación e implementación de políticas públicas que realmente contribuyan a la salud de la familia gestante y repercutan positivamente en la accesibilidad, oportunidad, pertinencia, seguridad y calidad en la atención que se brinda a las madres en su proceso de parto. Es decir, políticas que defiendan el proceso del parto, en un contexto en el que la salud sea un derecho y no una mercancía.

Generar leyes que tipifiquen la violencia obstétrica como un delito y sancionen a quienes ejerzan esta forma de violencia contra la mujer.

El personal que atiende a las madres debe empoderarse de la atención humanística y transpersonal de este proceso del parto y evitar así caer en una atención de la maternidad centrada en la mercantilización y en la medicalización de la vida. 


\section{Agradecimientos}

Artículo derivado de la investigación para optar el título de magíster en Salud Pública. Este proyecto fue financiado por el programa CODI-SOCIAL de la Universidad de Antioquia, bajo el contrato N. ${ }^{\circ}$ INV 514-16 Fecha: febrero del 2016 - febrero del 2018.

\section{Referencias bibliográficas}

1. Organización de las Naciones Unidas. Informe de la Conferencia Internacional sobre la Población y el Desarrollo [Internet]. El Cairo: OMS; 1994 [citado 2015 abr. 3] [194 páginas]. Disponible en: https://www.unfpa.org/w ebdav/site/global/shared/documents/publications/2004/icpd_spa.pdf

2. Colombia Ministerio de Salud y de la Protección Social. Plan Decenal de Salud Pública 2012-2021. Bogotá: El Ministerio.

3. Schutt-Aine J, Maddaleno M. Salud sexual y desarrollo de adolescentes y jóvenes en las Américas: implicaciones en programas y políticas [Internet]. OPS; 2003 [citado 2015 abr. 4] [73 páginas]. Disponible en: https://www.pah o.org/mex/index.php?option=com_docman\&task=doc_details\&gid=505\&Itemid=383

4. Organización de las Naciones Unidas. Declaración del Milenio. Nueva York, 8 de septiembre del 2000 [Internet] [citado 2014 sept. 15] [10 páginas]. Disponible en: https://www.un.org/spanish/milenio/ares552.pdf

5. Organización de las Naciones Unidas. Objetivos de Desarrollo Sostenible [Internet]. PNUD. 2015. [Internet] [citado 2015 oct. 30] [2 páginas]. Disponible en: https://www.un.org/sustainabledevelopment/es/objetivos-d e-desarrollo-sostenible/

6. Organización Mundial de la Salud - OMS. Mejorar la salud materna [Internet]. Ginebra: OMS; 2013 [citado 2015 oct. 30]. [2 páginas]. Disponible en: https://www.who.int/topics/millennium_development_goals/maternal_ health/es/

7. Álvarez C, Vargas M. Guía de atención del parto. Ministerio de la Protección Social [Internet]. 2006 [citado 2015 nov. 20] [623 páginas]. Disponible en: https://www.nacer.udea.edu.co/pdf/libros/guiamps/guias20.pdf

8. Organización Mundial de la Salud. Prevención y erradicación de la falta de respeto y el maltrato durante la atención del parto en centros de salud. Declaración de la OMS. Ginebra: OMS; 2014. p. 6-9.

9. Valdez-Santiago R, Hidalgo-Solano E, Mojarro-Iñiguez M. Nueva evidencia a un viejo problema: el abuso de las mujeres en las salas de parto. Revista Conamed. 2013;18(1):14-20.

10. Schwarz P. La maternidad tomada. Ginecólogos, obstetras y mujeres en interacción. Discursos y prácticas en la clase media. Rev. Crítica Social Argumentos. 2010;11:110-35.

11. Laurell AC. La segunda reforma de salud. Aseguramiento y compra-venta de servicios. Salud Colect. 2010;6(2):136-48.

12. Aguiar JM, D’Oliveira AFPL, Schraiber LB. Violência institucional, autoridade médica e poder nas maternidades sob a ótica dos profissionais de saúde. Cad Saúde Publica. 2013;29(11):2287-96.

13. Belli LF. La violencia obstétrica: otra forma de violación a los derechos humanos. Red Bioet UNESCO. 2013;1(7):25-34.

14. Thomson G, Downe S. Wide - Ning the trauma discourse: The link between childbirth and experiences of abuse. J Psychosom Obstet Gynaecol [Internet]. 2008;29(4):268-73 [citado 2015 dic. 20] [5 páginas]. Disponible en: https://www.tandfonline.com/doi/full/10.1080/01674820802545453

15. Strauss A, Corbin J. Bases de la investigación cualitativa. Técnicas y procedimientos para desarrollar la Teoría Fundamentada. Medellín: Universidad de Antioquia; 2003.

16. Galeano Marín ME. Teoría fundada: arte o ciencia. Estrategias de investigación social cualitativa: El giro de la mirada. Medellín: La Carreta Editores; 2004. p. 161-85.

17. Erwin Esaú AS, Rueda Arenas JF. La saturación teórica en la teoría fundamentada: su delimitación en el análisis de trayectorias de vida de víctimas del desplazamiento forzado en Colombia. Rev Colomb Sociología [Internet]. 
2013;36(2):93-104 [citado 2015 nov. 1] [11 páginas]. Disponible en: https://www.bdigital.unal.edu.co/38643 /1/41641-189266-1-PB.pdf

18. Cuesta M, Herrero FJ. Introducción al muestreo [Internet]. Oviedo: Universidad Andrés Bello; 2004 [citado 2015 nov. 1] [9 páginas]. Disponible en: https://www.mey.cl/apuntes/muestrasunab.pdf

19. Pérez Lugo JE. Sistemas de salud en América Latina: entre aciertos y desaciertos. Omnia. 2012;18:148-62

20. Kickbusch I. Los aspectos sociales de la educación. Educación para la salud Promoción y educación para la salud. Hamburgo: Unesco; 1997.

21. Rubio León DC. Prácticas institucionales de atención en salud del personal encargado de la prevención del cáncer de cuello uterino en una unidad prestadora de servicios de salud de la red pública hospitalaria de Medellín, Colombia Rev Facultad Nacional de Salud Pública. 2012;30:80-1.

22. Montoya JD. Por ahora, le cortan cordón umbilical a la Clínica León XIII. El Colombiano Sec Histórico [Internet]. Medellín; 2011 Oct 4; [citado 2016 feb. 22]. [5 páginas]. Disponible en: https://www.elcolombiano.com/hist orico/por_ahora_le_cortan_cordon_umbilical_a_la_leon_xiii-CYEC_152752

23. Cano CM. Atención ágil de maternas está en riesgo en Medellín. El Tiempo [Internet]. Bogotá; 2012 Jun 14 [citado 2016 feb. 22]. [4 páginas]. Disponible en: https://www.eltiempo.com/archivo/documento/CMS-11945121

24. Santos M. Por otra globalización: Del pensamiento único a la conciencia universal. Bogotá: Convenio Andrés Bello; 2004.

25. Odent M. El bebé es un mamífero. Buenos Aires: Madreselva; 2011.

26. Banco Mundial. Invertir en salud: Informe sobre el Desarrollo Mundial. Washington D. C.: Banco Mundial; 1993.

27. Almeida C. Reforma del sector salud y equidad en América Latina y el Caribe: conceptos, agenda, modelos y algunos resultados de implementación. Rev Gerencia y Políticas de Salud. 2005;4(9):6-60.

28. Odent M. El nacimiento en la era del plástico. Buenos Aires: Creavida; 2011.

29. Bourdieu P, Passeron JC. La reproducción: Elementos para una teoría de la enseñanza. Tercera edición. Mexico, D. F.: Laia; 1996.

30. Martin E. A mulher no corpo. Uma análise cultural da reproduçáo. Río de Janeiro: Garamond; 2006.

31. García-Jordá D, Díaz-Bernal Z, Acosta Álamo M. El nacimiento en Cuba: análisis de la experiencia del parto medicalizado desde una perspectiva antropológica. Rev Cien y Saude Colet [Internet] 2012;17 (7):1893-902 [citado 2017 jun. 20]. Disponible en: https://www.scielo.br/pdf/csc/v17n7/29.pdf

32. Bermejo JC. Salir de la noche: Por una enfermería humanizada. Maliaño, España: Sal Terrae; 1999.

33. Boff L. El cuidado esencial. Madrid: Trotta; 2002.

34. Camacaro-Cuevas M. Patologizando lo natural, naturalizando lo patológico... improntas de la praxis obstétrica. Rev Venez Estud La Mujer. 2009;14:147-62.

\section{Licencia Creative Commons CC BY 4.0}

Para citar este artículo: Arango Urrea JD, Molina Berrío DP, Mejía Merino CM, Zapata LF. La atención a las madres durante el proceso de parto en algunos servicios de salud de la ciudad de Medellín: un acontecimiento enmarcado en el neoliberalismo y la mercantilización de la vida. Rev. Gerenc. Polit. Salud. 2018;17(35). https://doi.org/10.11144/Javeriana.rgps17-35.amdp 